\title{
Phylogenomics of an extra-Antarctic notothenioid radiation reveals a previously unrecognized lineage and diffuse species boundaries
}

Santiago G. Ceballos ${ }^{1,2,3^{*}}$ (D, Marius Roesti ${ }^{3,4}$, Michael Matschiner ${ }^{3,5}$, Daniel A. Fernández ${ }^{1,2}$, Malte Damerau ${ }^{6}$, Reinhold Hanel ${ }^{6}$ and Walter Salzburger ${ }^{3}$

\begin{abstract}
Background: The impressive adaptive radiation of notothenioid fishes in Antarctic waters is generally thought to have been facilitated by an evolutionary key innovation, antifreeze glycoproteins, permitting the rapid evolution of more than 120 species subsequent to the Antarctic glaciation. By way of contrast, the second-most species-rich notothenioid genus, Patagonotothen, which is nested within the Antarctic clade of Notothenioidei, is almost exclusively found in the non-Antarctic waters of Patagonia. While the drivers of the diversification of Patagonotothen are currently unknown, they are unlikely to be related to antifreeze glycoproteins, given that water temperatures in Patagonia are well above freezing point. Here we performed a phylogenetic analysis based on genome-wide single nucleotide polymorphisms (SNPs) derived from restriction site-associated DNA sequencing (RADseq) in a total of twelve Patagonotothen species.
\end{abstract}

Results: We present a well-supported, time-calibrated phylogenetic hypothesis including closely and distantly related outgroups, confirming the monophyly of the genus Patagonotothen with an origin approximately 3 million years ago and the paraphyly of both the sister genus Lepidonotothen and the family Notothenidae. Our phylogenomic and population genetic analyses highlight a previously unrecognized linage and provide evidence for shared genetic variation between some closely related species. We also provide a mitochondrial phylogeny showing mitonuclear discordance.

Conclusions: Based on a combination of phylogenomic and population genomic approaches, we provide evidence for the existence of a new, potentially cryptic, Patagonotothen species, and demonstrate that genetic boundaries between some closely related species are diffuse, likely due to recent introgression and/or incomplete linage sorting. The detected mitonuclear discordance highlights the limitations of relying on a single locus for species barcoding. In addition, our time-calibrated phylogenetic hypothesis shows that the early burst of diversification roughly coincides with the onset of the intensification of Quaternary glacial cycles and that the rate of species accumulation may have been stepwise rather than constant. Our phylogenetic framework not only advances our understanding of the origin of a high-latitude marine radiation, but also provides the basis for the study of the ecology and life history of the genus Patagonotothen, as well as for their conservation and commercial management.

Keywords: RAD, Adaptive radiation, Patagonotothen, Cryptic species

\footnotetext{
* Correspondence: sgceballos@gmail.com

${ }^{1}$ Instituto de Ciencias Polares, Ambiente y Recursos Naturales (ICPA),

Universidad Nacional de Tierra del Fuego (UNTDF), Fuegia Basket 251,

V9410BXE Ushuaia, Argentina

${ }^{2}$ Centro Austral de Investigaciones Científicas (CADIC-CONICET), Bernardo A

Houssay 200, V9410BXE Ushuaia, Ushuaia, Tierra del Fuego, Argentina

Full list of author information is available at the end of the article
}

(c) The Author(s). 2019 Open Access This article is distributed under the terms of the Creative Commons Attribution 4.0 International License (http://creativecommons.org/licenses/by/4.0/), which permits unrestricted use, distribution, and reproduction in any medium, provided you give appropriate credit to the original author(s) and the source, provide a link to the Creative Commons license, and indicate if changes were made. The Creative Commons Public Domain Dedication waiver (http://creativecommons.org/publicdomain/zero/1.0/) applies to the data made available in this article, unless otherwise stated. 


\section{Background}

Species radiations, that are the rapid evolution of taxonomic, ecological and morphological disparity within a clade, have long been recognized as essential models to study organismal diversification [1]. The most extensive case of a fish radiation in the marine environment is represented by the Antarctic clade of the teleost suborder Notothenioidei that has diversified into over 120 species [2-4]. Notothenioids dominate the fish fauna on the Antarctic continental shelves, where they comprise approximately $77 \%$ of the fish species diversity and more than $90 \%$ of fish biomass [2]. It is assumed that the evolutionary innovation of antifreeze glycoproteins (AFGPs) was key for the successful colonization of cold water niches, which in turn triggered the adaptive radiation of the Antarctic notothenioids [4-8]. It has further been proposed that the Antarctic notothenioid radiation was shaped by paleoclimatic changes that resulted in continuous ecological divergence into recurrently opening niches $[6,9]$. Today, the bulk of notothenioid species inhabit the area south of the Antarctic Polar Front.

The notothenioid genus Patagonotothen, however, is found almost exclusively in non-Antarctic waters. It is nested within the Antarctic notothenioid clade and represents the second-most species-rich genus of Notothenioidei with 15 recognized species to date (Eastman and Eakin available at https://people.ohio.edu/eastman/; version Dec. 15, 2016), only surpassed in taxonomic diversity by the Antarctic genus Pogonophryne with 24 species. All Patagonotothen species occur in marine waters around the southern part of South America (Patagonia), the only exception being $P$. guntheri, which has a trans-Antarctic Polar Front range extending from the southern Patagonian Shelf to the Shag Rocks Shelf [10, 11]. Morphological analyses indicate that $P$. guntheri is a derived species within the genus, suggesting that it dispersed southward secondarily $[10,12]$ and that, therefore, the initial Patagonotothen radiation most likely occurred in non-Antarctic waters. The age of the most recent common ancestor of Patagonotothen has been estimated to be around $5 \mathrm{Ma}$ [6], making it a relatively recent radiation. The exact drivers of this radiation remain unknown, but are unlikely to be related to the putative evolutionary key innovation of notothenioids, the AFGPs, since the temperature of Patagonian waters is usually well above freezing point [13]. Furthermore, it has been shown that at least some Patagonotothen species have secondarily lost the ability to produce AFGPs $[4,7,14]$. Interestingly, an unrelated but similarly species-rich radiation has occurred in the same geographic region in the mollusc genus Nacella. In this case, it has been proposed that the currently overlapping distribution of several closely related Nacella species constitutes a secondary contact scenario after allopatric speciation, or incipient separation in different refugia during glaciation cycles followed by geographical re-expansion and ecological separation [15]. Whether the same processes also underlie the Patagonotothen radiation remains unknown.

In addition to extrinsic environmental factors, intrinsic factors (such as dispersal ability, genetic architecture or chromosomal instability; see [3]) specific to Patagonotothen might have played a role in the diversification of this clade. This is suggested, for example, by their comparison with the evolutionary history of Eleginops maclovinus, another notothenioid species occurring along the Patagonian coast. E. maclovinus is the only representative of the family Eleginopsidae and has an estimated divergence time from the Antarctic clade of around $40 \mathrm{Ma}$ [5]. This species shows strong genetic footprints of past glacial cycles $[16,17]$; however, only weak population structure is found along the Pacific and Atlantic Patagonian coast $[16,17]$. Thus, while E. maclovinus has remained a single taxonomic unit for an extended period of time, Patagonotothen have diversified into more than a dozen of species in a relatively short period of time in the same geographic region.

The Patagonotothen species are of great abundance throughout the Patagonian shelf [18] and play an important role in the trophic ecology of this region: They consume a variety of benthic and supra-benthic invertebrates $[10,19,20]$ and are prey to almost all medium to large-sized fish predators including hakes (Merluccius spp.), toothfish (Dissostichus eleginoides), kingclip (Genypterus blacodes), redcod (Salilota australis) and rajids [21]. Some Patagonotothen species are or have been subject to extensive exploitation (especially $P$. ramsayi and $P$. guntheri) and represent a significant proportion of the by-catch of bottom trawl fisheries [22, 23]. Despite the ecological and economic importance of Patagonotothen, the genus has been little explored with respect to its taxonomy and evolutionary history. One problem is that some of the species are very similar morphologically, making species identification difficult [24-26]. Knowing the taxonomic status, and the number of independent evolutionary units in that genus, is important not only for understanding the Patagonotothen radiation, but also to study their ecology and life history, as well as for conservation and management purposes. In addition, the evolutionary history of Patagonotothen - that is, the colonization of warmer and thermally more unstable waters from Antarctic ancestors and the subsequent diversification - resembles, at least to some extent, a possible scenario that could apply to other notothenioids in the light of global warming and the associated rise in water temperature in the Southern Ocean.

Most available molecular phylogenetic hypotheses that aimed at resolving the evolutionary relationships among notothenioids and that include Patagonotothen species 
are based on a small number of representatives of this genus and on mitochondrial and/or few nuclear markers, generally yielding low to moderate support for internal phylogenetic nodes $[6,27]$. In this study, we report a new time-calibrated phylogenetic hypothesis for Patagonotothen that is based on genome-wide single nucleotide polymorphisms (SNPs) identified through restriction site-associated DNA sequencing (RADseq) in twelve Patagonotothen species, including a putative new species. We also assessed the level of differentiation between closely related species within the genus Patagonotothen, applying a population genomic approach.

\section{Results}

\section{De novo assembly of RAD loci}

We conducted a de novo assembly of RAD loci with the software Stacks, version 1.41 [28] and assessed the performance of parameters following the protocol suggested in reference [29] (see Methods for more details). Briefly, we varied parameters $M$ (distance allowed between stacks) and $\mathrm{n}$ (distance allowed between catalogue loci) from 1 to 6 (fixing $M=n$ ), and plotted the number of loci shared by at least $80 \%$ of a subset of ten samples (Additional file 1). Considering that, for our data set, the number of widely shared loci plateaus starts at about $M$ $=3$, we retained this value for the main analysis. In addition, preliminary analyses using only a clade including two replicated samples showed a clustering according to species identity and replicated samples clustered together tightly in a Neighbour-Joining (NJ) tree (not shown) demonstrating that the de novo assembly (with parameters $\mathrm{M}=n=3$ ) performed with the Stacks pipeline generated robust results.

In the whole set of samples, we obtained $3.9 \pm 1.5$ million quality-filtered Illumina reads per individual. With these raw data the de novo assembly generated an average of $55,871 \pm 8520$ loci per individual with a stack depth of $59 \pm 20$.

\section{Species assignment and early diversification}

To test for phylogenetic structuring according to morphological species identification, and to evaluate the use of RADseq to resolve early cladogenetic events in this radiation, we first performed a Maximum-Likelihood (ML) phylogenetic analysis using all samples and including all outgroups (Table 1). Allowing for no more than $5 \%$ missing data, this analysis was based on 11,804 SNPs across 1682 RAD loci. All samples from the same species formed monophyletic groups as expected, except for three species of the genus Patagonotothen: P. cornucola, $P$. guntheri and $P$. ramsayi (Additional file 2 and Fig. 1; see following sections for further details). The reconstructed phylogeny supports the paraphyly of the family Nototheniidae as the position of $N$. coriiceps is closer to
$H$. bispinis (family Harpagiferidae) than to other members of the Nototheniidae (Fig. 1). The paraphyly of the genus Lepidonotothen is also supported, with $L$. kempi and $L$. squamifrons being more closely related to the genus Patagonotothen (Fig. 1) than L. larseni and $L$. nudifrons. Our finding of paraphyly of both Nototheniidae and Lepidonotothen is in agreement with previous phylogenetic studies of notothenioids based on smaller data sets $[5,6,27,30]$.

\section{ML phylogeny of Lepidonotothen and Patagonotothen}

To analyze the more recent speciation events, we performed a phylogenetic analysis with a separate data set excluding the distant outgroups E. maclovinus, $H$. bispinis and $N$. coriiceps to maximize the recovered number of loci and SNPs and thus to improve the resolution of the tree. In this case, Stacks recovered 2914 RAD loci across samples, harbouring a total of 18,485 SNPs $(2.5 \%$ missing data allowed). We performed a ML phylogenetic analysis with all Trematomus (these were used as outgroup), Lepidonotothen and Patagonotothen samples ( $n=$ 87) (Fig. 2 and Additional file 3), and recovered strong bootstrap support (>95) for most nodes in the resulting phylogeny. This analysis resulted in the following four key observations with implications for species delimitation:

(I). Evidence for a new Patagonotothen species: samples morphologically identified as $P$. cornucola (Richardson 1844) [31] following Brickle et al. [24] formed two divergent reciprocally monophyletic groups. These groups were also recovered using mtDNA (see below). We consider this to be good evidence for the presence of an unrecognized Patagonothen species, given that the level of divergence is comparable to the one observed between other well-established Patagonotothen species (Fig. 2). We therefore named the clade comprising the samples from the eastern limit of the Beagle Channel Patagonotothen cf. cornucola.

(II). The samples from $P$. brevicauda formed a monophyletic group nested within the paraphyletic P. guntheri clade.

(III). The samples of $P$. wiltoni formed a monophyletic group nested within the paraphyletic $P$. ramsayi clade.

(IV). Despite the close morphological similarity of $L$. kempi and L. squamifrons [32, 33], samples assigned morphologically to these species formed two reciprocally monophyletic groups.

In order to verify the paraphyly observed in P. guntheri and $P$. ramsayi with a model accounting for Incomplete Linage Sorting (ILS) we implemented a PolymorphismAware Phylogenetic Model. In the resulting topology both species also appear as paraphyletic (Additional file 4). 
Table 1 Species included in phylogenetic analyses, geographic origin of specimens, sample size and collecting depth for species of Patagonotothen

\begin{tabular}{|c|c|c|c|}
\hline Species & Origin (Sample size) & COI GenBank & Collecting depth (meters) \\
\hline Eleginops maclovinus & Beagle Channel (4) & $N / A^{*}$ & - \\
\hline Harpagifer bispinis & Beagle Channel (3) & N/A & - \\
\hline Notothenia coriiceps & South Shetland Islands (2) & N/A & - \\
\hline Trematomus eulepidotus & South Shetland Islands (3) & MG770024-MG770025 & - \\
\hline Lepidonotothen larseni & South Shetland Islands (3) & MG770008-MG770010 & - \\
\hline Lepidonotothen nudifrons & South Shetland Islands (4) & MG770011-MG770014 & - \\
\hline Lepidonotothen squamifrons & Shag Rocks (5) & MG770015-MG770019 & - \\
\hline \multirow[t]{3}{*}{ Lepidonotothen kempi } & Shag Rocks (1) & N/A & - \\
\hline & Bouvet Island (2) & MG770020-MG770021 MG770022- & - \\
\hline & South Orkney Islands (2) & MG770023 & - \\
\hline Patagonotothen sima & Beagle Channel (6) & MG769992-MG769995 & $<1$ \\
\hline Patagonotothen jordani & Atlantic Patagonian Shelf (4) & MG770004-MG770007 & 36 \\
\hline Patagonotothen elegans & Atlantic Patagonian Shelf (3) & MG770000-MG770003 & 57 \\
\hline \multirow[t]{2}{*}{ Patagonotothen cornucola } & Beagle Channel (2) & MG769996-MG769997 & $<1$ \\
\hline & Atlantic coast of TDF** (2) & MG769998-MG769999 & $<1$ \\
\hline Patagonotothen cf. cornucola & Eastern limit of Beagle Channel (3) & MG770026-MG770028 & 30 \\
\hline Patagonotothen canina & Atlantic Patagonian Shelf (3) & MG769956-MG769959 & 77 \\
\hline Patagonotothen tessellata & Beagle Channel (7) & MG769948-MG769955 & $<1$ \\
\hline Patagonotothen trigramma & Beagle Channel (1) & N/A & 6 \\
\hline \multirow[t]{2}{*}{ Patagonotothen guntheri } & Atlantic Patagonian Shelf (5) & MG769960-MG769962 & $100-128$ \\
\hline & Shag Rocks (3) & MG769963-MG769964 & 309 \\
\hline Patagonotothen brevicauda & Atlantic Patagonian Shelf (5) & MG769965-MG769969 & $52-84$ \\
\hline \multirow[t]{2}{*}{ Patagonotothen ramsayi } & Atlantic Patagonian Shelf (12) & MG769970-MG769983 & $60-100$ \\
\hline & East of Burwood Bank (3) & MG769976-MG769978 & 309 \\
\hline Patagonotothen wiltoni & Beagle Channel (8) & MG769984-MG769991 & $6-10$ \\
\hline
\end{tabular}

*Non Available; **Tierra del Fuego

GenBank accession numbers for mitochondrial COI sequences are indicated

Analysis of closely related species pairs using STRUCTURE To further investigate differentiation between the closely related pairs of species $P$. guntheri $-P$. brevicauda, $P$. ramsayi - P. wiltoni, and L. squamifrons - L. kempi, we generated input files for STRUCTURE using the population.map.pl component of the Stacks pipeline, including a single randomly selected SNP from each RAD locus and tolerating no missing data. The results of the STRUCTURE analyses are shown in Fig. 3. For the species pairs $P$. guntheri - P. brevicauda and $P$. ramsayi $P$. wiltoni, the most likely number of genetic clusters (K) was identified to be 2 or 3 . We have considered both since biological meaningful information was observed. When assuming two clusters $(\mathrm{K}=2)$ within the $P$. brevicauda - P. guntheri pair some samples of P. guntheri are identical to $P$. brevicauda; however, when assuming $\mathrm{K}=$ 3 , one cluster correspond exclusively to the P. brevicauda samples and the other two to the P. guntheri samples, with one individual showing an admixed genotype.
A substantial level of genetic admixture is also present in the $P$. wiltoni $-P$. ramsayi clade when assuming $\mathrm{K}=$ 2 , although the two species appear clearly differentiated. Further genetic heterogeneity is revealed within $P$. ramsayi when assuming three genetic clusters $(\mathrm{K}=3)$. For the $L$. kempi - L. squamifrons pair, the most likely value of the number of genetic clusters was 2. STRUCTURE was able to completely separate the species.

\section{Time-calibrated phylogeny inference with SNAPP}

Three samples per species were selected for the SNAPP analysis except for $P$. trigramma from which only one specimen was available. For the species pairs $P$. guntheri - P. brevicauda and $P$. ramsayi $-P$. wiltoni, we considered the ML tree and the STRUCTURE results to select samples in order to capture most of the diversity within the species pairs. With these conditions Stacks generated an output file with 2778 SNPs. The SNAPP tree topology (Fig. 4a) was identical to the ML topology (Fig. 2) 


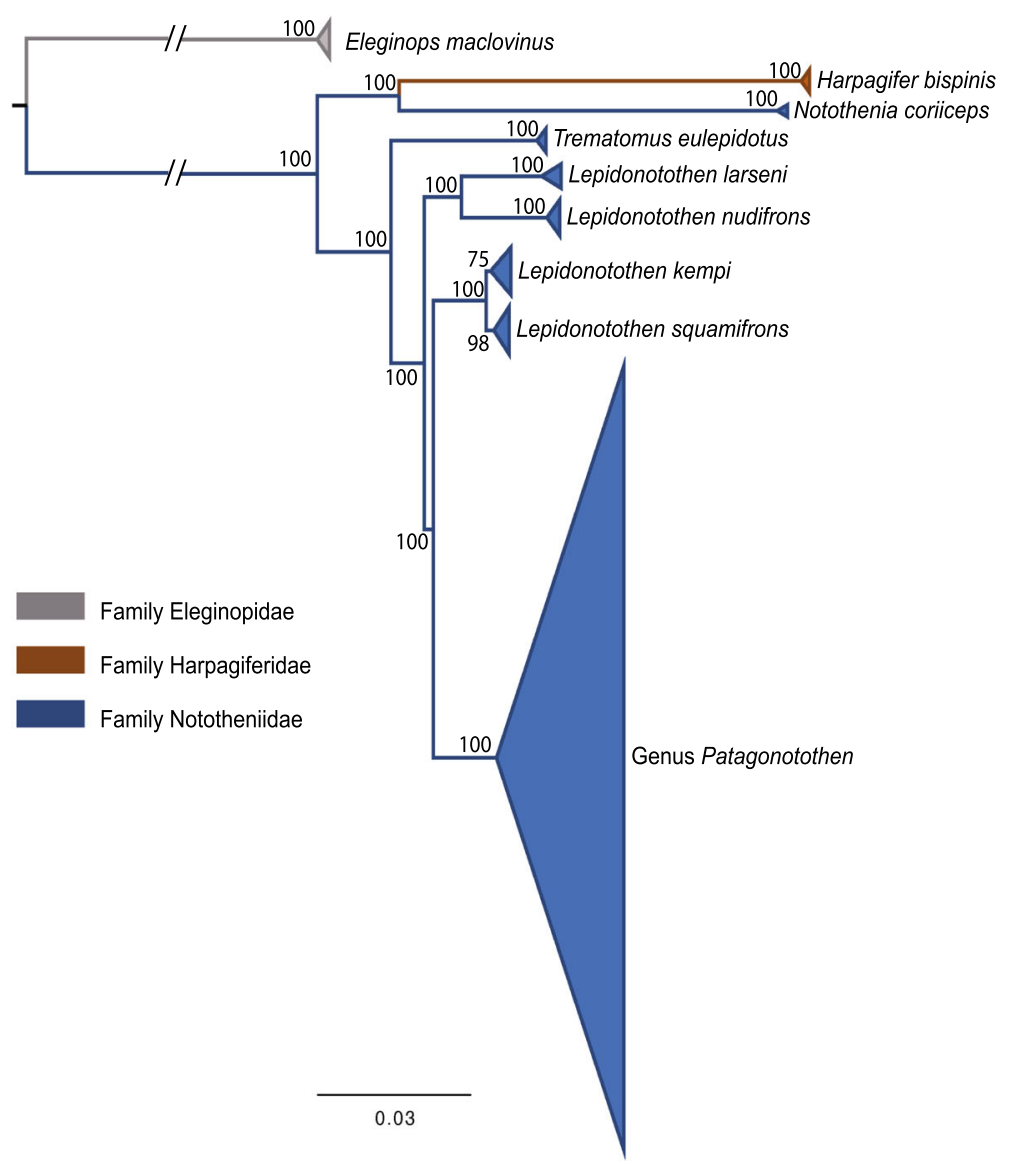

Fig. 1 Maximum-Likelihood tree based on 11,804 SNPs from 1682 RAD loci including distant outgroups to assess early diversification in nototheniods. Individuals from the same species in outgroups and from all Patagonotothen species have been collapsed for clarity (see full tree in Additional file 1: Figure S1). Node labels represent bootstrap support values as obtained with RAxML

except for the relative positions of $P$. sima and $P$. jordani. Whereas $P$. jordani diverges earlier than $P$. sima in the ML tree, these two species appeared as sister taxa in the SNAPP phylogeny. The node supporting their sister-group relationship in the SNAPP phylogeny had a relatively low posterior support (0.703), probably related to the short internal branch leading to this speciation event. Figure $4 \mathrm{~b}$ depicts the rate of speciation events and shows a remarkable period of more than one million years without reconstructed speciation events between approximately $2.3 \mathrm{Ma}$ to $1.2 \mathrm{Ma}$ ago.

\section{Mitochondrial locus}

Maximum-Likelihood and the NJ reconstructions of the Cytochrome Oxidase I (COI) phylogeny resulted in a similar topology (Fig. 5 and Additional files 5 and 6); however, many nodes in the ML mitochondrial phylogeny had low bootstrap support $(<70)$. The mitochondrial locus failed to resolve the taxonomic identity of $P$. brevicauda and $P$. guntheri since the corresponding haplotypes did not form two reciprocally monophyletic clades. Despite a relatively high $F_{\mathrm{ST}}$ between $P$. wiltoni and $P$. ramsayi, we found three haplotypes shared between them (Fig. 5, Table 2). More specifically, two individuals of $P$. ramsayi had a COI haplotype found in most $P$. wiltoni samples, and the opposite was true for one $P$. wiltoni individual. In addition, the mitochondrial haplotype genealogy and phylogeny (Fig. 5 and Additional files 5 and 6) do not make clear that $P$. wiltoni and $P$. ramsayi are more closely related to one another than to either $P$. guntheri or $P$. brevicauda as is revealed by the nuclear phylogeny. On the other hand, $P$. cornucola and $P$. cf. cornucola still appeared as sister species and were well discriminated with the mitochondrial locus in accordance with the nuclear analysis. Regarding the Lepidonotothen species pair L. kempi - L. squamifrons, the mitochondrial locus was unable to resolve species identity in contrast to the RADseq data.

\section{Discussion}

The main goal of this work was to generate a high-resolution phylogenetic framework for the genus Patagonotothen to better characterize the diversity of 


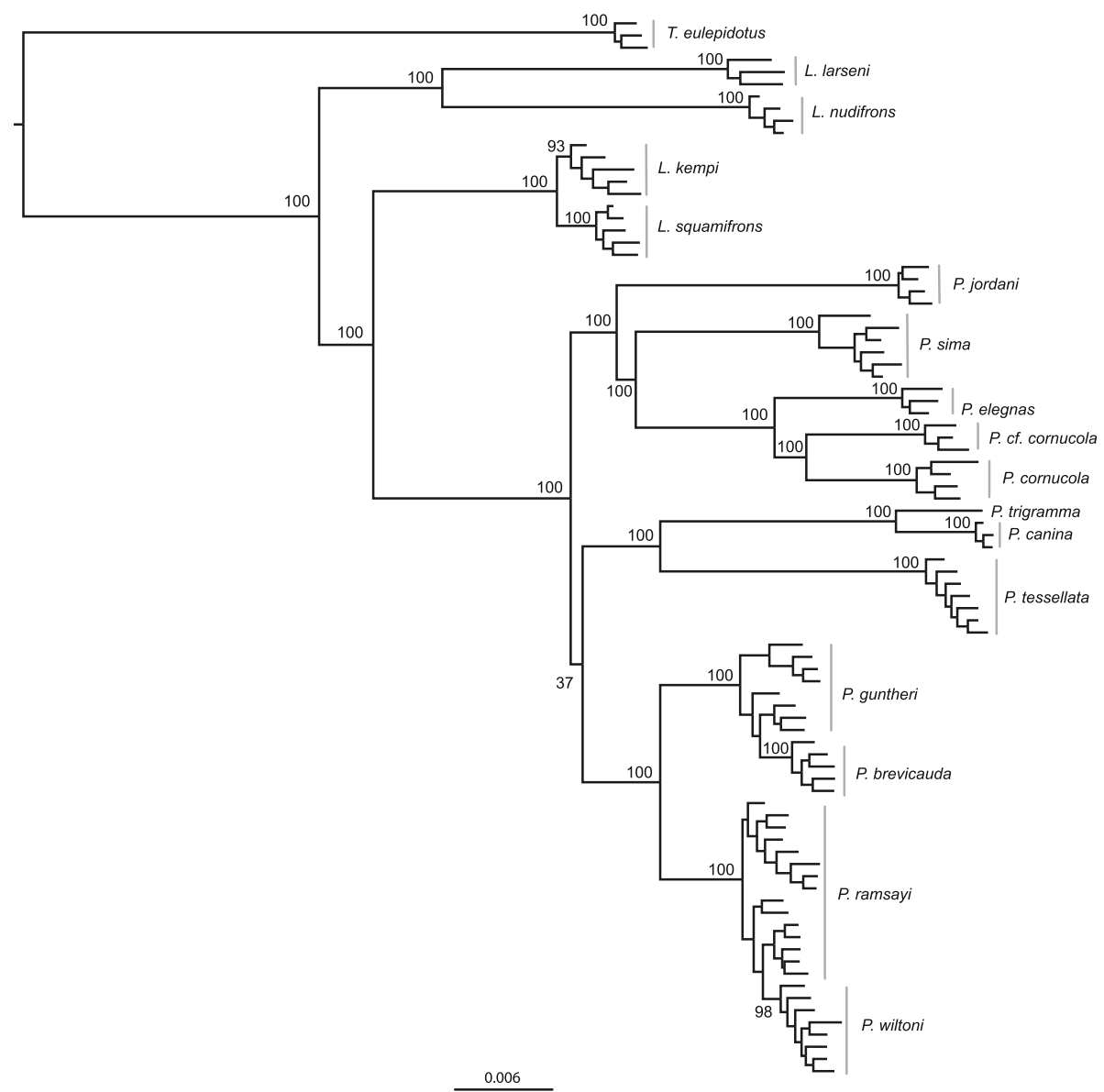

Fig. 2 Maximum-Likelihood tree based on 18,485 SNPs from 2914 RAD loci. Distant outgroups (E. maclovinus, H. harpagifer and N. coriiceps) were excluded in order to maximize the recovered number of loci and SNPs. Node labels represent bootstrap support values as obtained with RAxML. Bootstrap support values within species were omitted for clarity (See full tree in Additional file 2: Figure S2)

this group and to obtain insights into the processes that drove the radiation of this genus. The parameters used for the de novo assembly and filtering of loci performed well as demonstrated by the analysis including replicated samples (following the method suggested in reference [34]) and by the plot of loci shared by $80 \%$ of samples (Additional file 1), but also by the general agreement of the results with previous molecular phylogenies that show, for example, the paraphyly of the family Nototheniidae and the genus Lepidonotothen $[5,6,27,30]$. At a finer taxonomic resolution, our phylogenetic results are generally in agreement with morphological differences, the geographic origin and/or the mitochondrial haplogroup of the samples.

\section{Taxonomic implications}

\section{Previously unrecognized linage}

The individuals morphologically identified as $P$. cornucola (Richardson 1844 [31]) following Brickle et al. (2005) [24] formed two well-differentiated groups in our analyses, both based on genome-wide SNPs and a mitochondrial marker (Figs. 2 and 6). These groups have an estimated divergence time of $\sim 1 \mathrm{Ma}$ (Fig. 4). The level of genetic divergence between these two lineages (Table 2) is comparable to the divergence of any of them to the next-closest species $P$. elegans, or between other morphologically well-differentiated pairs of species like $P$. ramsayi $-P$. guntheri or $P$. canina $-P$. trigramma (Fig. 4a). In addition, geographic distance is unlikely to account for the separation of the two lineages because the samples of one clade were collected in the Beagle Channel (Bahía Golondrina, Ushuaia) and on the Atlantic coast of Tierra del Fuego (Auricosta), whereas the samples of the other clade were collected halfway between these two sampling points near the eastern limit of the Beagle Channel (Table 1 and Fig. 6). However, the individuals forming the former clade were caught at the intertidal zone, whereas the individuals of the second clade were caught at a depth of $30 \mathrm{~m}$. This difference in collecting depth between these clades suggests spatial divergence at an ecological scale between the two lineages. Together, our results strongly support the recognition of 


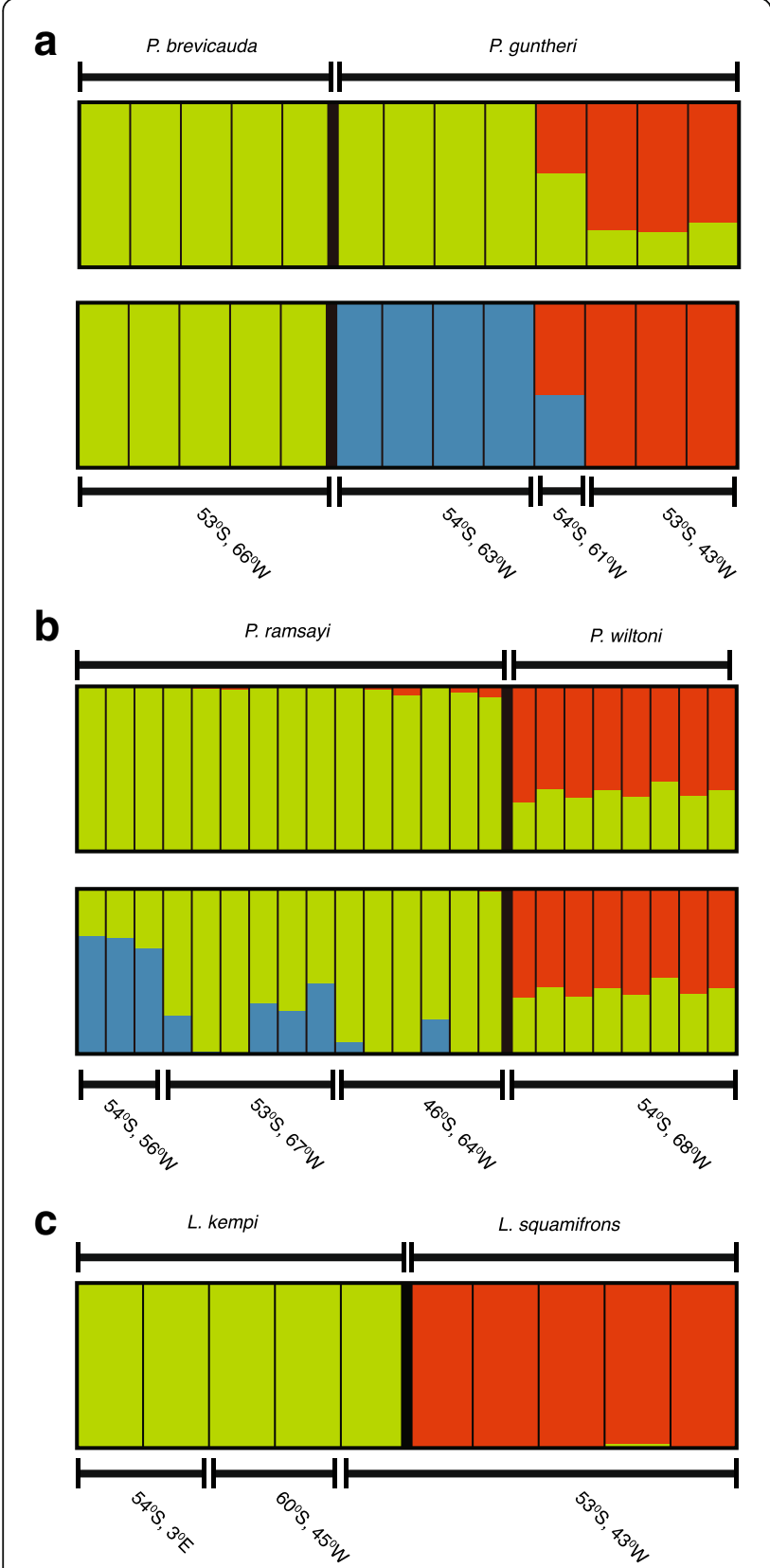

Fig. 3 Analysis of differentiation between closely related species pairs using the software STRUCTURE based on genomic SNPS obtained through RADseq and assuming two (top) or three (bottom) inferred clusters. Below the plots the geographic origin of samples is indicates in latitude and longitude coordinates. a $P$. breviauda / P. guntheri species-pair differentiation inferred from a total of 2466 SNPs. b P. ramsayi / P.wiltoni species-pair differentiation inferred from a total of 3458 SNPs. c L. kempi / L. squamifrons species-pair differentiation inferred from a total of 999 SNPs

the samples morphologically identified as $P$. cornucola as two genetically distinct, yet morphologically similar species.

Very little is known about the biology of P. cornucola. Most of the few studies carried out so far on $P$. cornucola have collected the individuals at the intertidal zone or in very shallow waters [19, 35-37]. In addition, the original description of the species by Richardson (1844) [31] states that specimens were collected "among the sea-weed that lines the shores of Cape Horn", suggesting that the individuals were fished at very shallow waters. Thus, we used the $P$. cornucola denomination for the clade formed by the samples caught at the intertidal zone of Tierra del Fuego and Patagonotothen cf. cornucola for the species collected at the eastern limit of the Beagle Channel. Future studies should attempt to identify morphological differences between these two species to determine whether they are indeed morphologically indistinguishable and could therefore be considered cryptic, as has been previously suggested for Antarctic notothenioids species within the genus Lepidonotothen [38]. Until a morphological description of Patagonotothen cf. cornucola becomes available, the two species can be diagnosed by their distinct COI haplotypes (Table 1 ).

\section{The P. guntheri - P. brevicauda species pair}

The taxonomic identity of these two closely related species could not be resolved with $\operatorname{mtDNA}\left(F_{\mathrm{ST}}=0\right)$ and little differentiation was found across all genome-wide SNPs (mean $F_{\mathrm{ST}}=0.027$ ). In addition, the specimens of $P$. guntheri did not form a monophyletic group in the ML phylogeny (Fig. 2) and the genetic clustering conformed more to geography than morphology (Fig. 3a and Fig. 6). This is supported for $K=2$ (STRUCTURE) in Fig. 3, where the Patagonian Shelf population of $P$. guntheri appeared to be closer to the P. brevicauda from the Patagonian Shelf than to the Shag Rock samples of $P$. guntheri. However, the single P. guntheri sample caught at Namuncurá - Burdwood Bank, which is geographically in between the other two sampling locations of P. guntheri, showed mixed membership (Fig. 3a and Fig. 6), suggesting a possible pattern of isolation by distance. Balushkin et al. (2000) [12] proposed that the Shag Rocks population of P. guntheri should be considered a different species ( $P$. shagensis). Our analyses of mitochondrial and nuclear markers rather argue for $P$. guntheri from Shag Rock to be a different geographic population, or a subspecies, rather than a different species, similar to the case of P. brevicauda.

Morphologically, P. brevicauda and P. guntheri are very similar. The main diagnostic morphologies allowing their distinction are the depth of the caudal peduncle and the number of gill rakers (Norman, 1937). It has been suggested that P. guntheri usually occupies deeper waters than $P$. brevicauda, thus providing as similar case to the one found in P. ramsayi and P. wiltoni (Norman, 1937). Different evolutionary scenarios may account for the pattern found for P. brevicauda and P. guntheri, and 


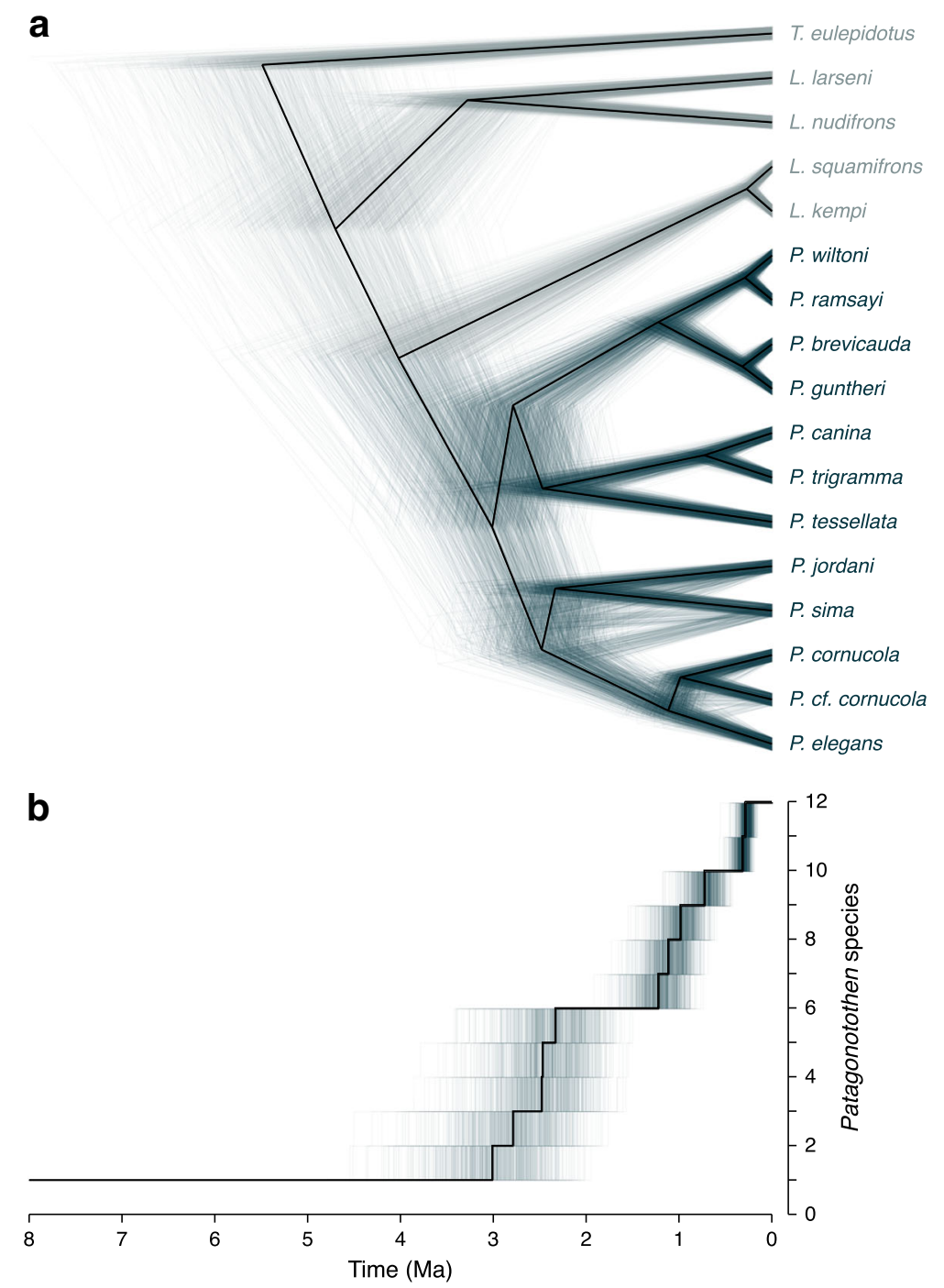

Fig. 4 a Time-calibrated phylogeny generated with the software SNAPP based on 2778 SNPs. Three samples were included per species in the analysis except for $P$. trigramma from which only one specimen was available. $\mathbf{b}$ Lineage accumulation though time for Patagonotothen species

only a broad population sampling could allow determining the genetic relationship between these two closely related species. Note that one of the P. guntheri samples (sample code: gun128 in the Additional files 5 and 6) has a mitochondrial haplotype that is closely related to the $P$. wiltoni mitochondrial clade; however, nuclear SNPs place this sample in a nested position within the $P$. guntheri clade, indicating that recent introgression may have occurred between $P$. wiltoni and P. guntheri.

\section{The $P$. wiltoni - $P$. ramsayi species pair}

We found a rather complex scenario in this species pair. While separated well at the mitochondrial marker $\left(F_{\mathrm{ST}}\right.$ $=0.63$ ), our genome-wide SNPs suggested a much weaker differentiation between $P$. wiltoni and $P$. ramsayi (mean $F_{\mathrm{ST}}=0.015$ ). In spite of the high $F_{\mathrm{ST}}$ value observed for mtDNA, both species share mitochondrial haplotypes (Fig. 5), suggesting past or ongoing hybridization. The STRUCTURE analysis also suggested a substantial level of shared genetic variation between the two species. In addition, the paraphyly of $P$. ramsayi as well as the heterogeneity observed with STRUCTURE (assuming $K=3$ ) suggested that the level of differentiation between some $P$. ramsayi specimens is comparable to the differentiation found between $P$. ramsayi and $P$. wiltoni. Divergence into separate refugia during glaciation, possibly followed by admixture during postglacial periods, may be a potential explanation for these patterns. The simplest explanation for the much greater differentiation at the mitochondrial marker may be related to the four times lower effective population size in mitochondrial sequences compared to nuclear loci. 


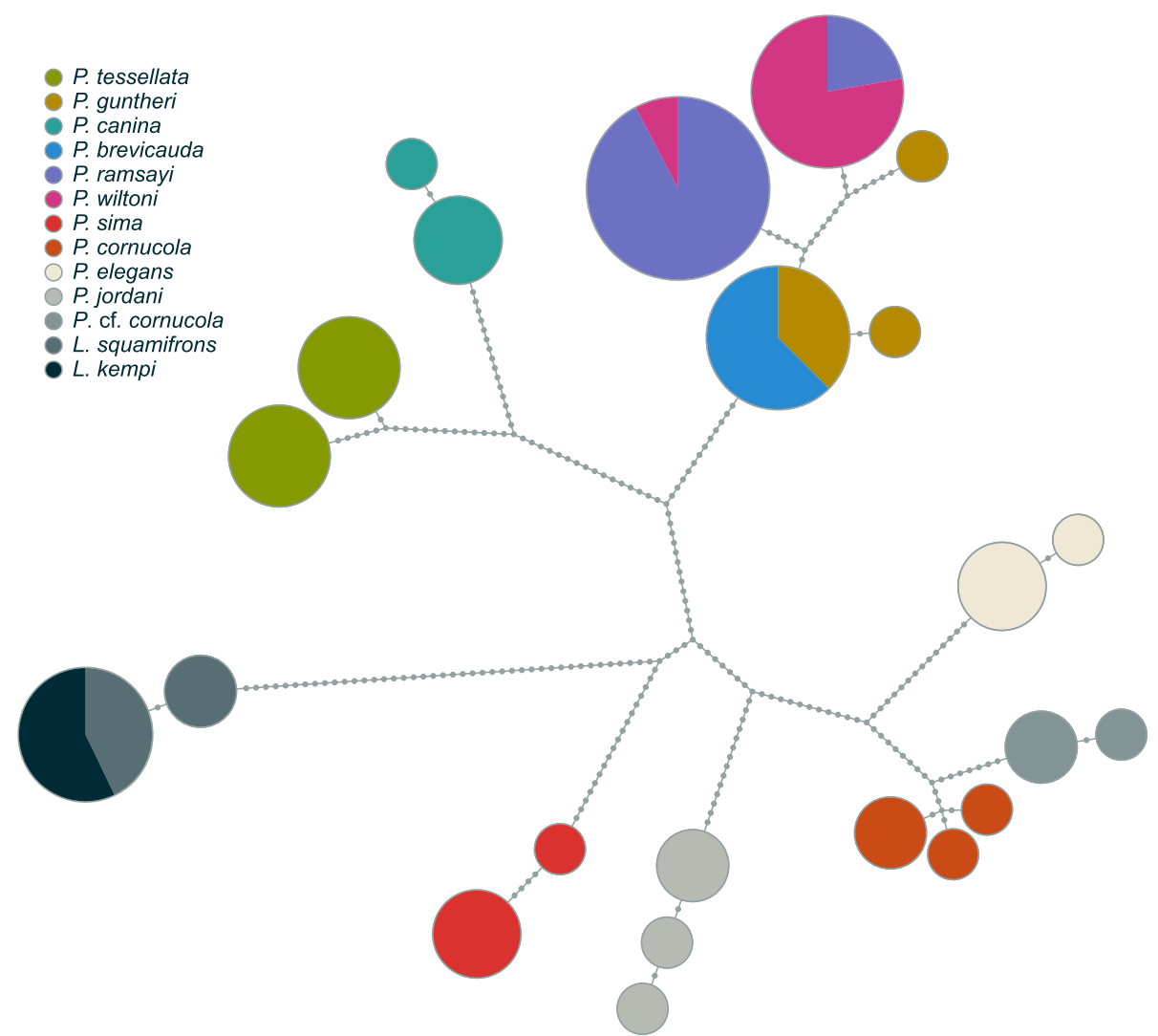

Fig. 5 Mitochondrial haplotype genealogy generated with the software Fitchi based on a maximum-likelihood tree (Additional file 3: Figure S3). Haplotypes differing by a single nucleotide were collapsed for clarity

From a morphological standpoint, $P$. wiltoni and $P$. ramsayi are very similar. $P$. wiltoni can be distinguished from $P$. ramsayi by the smooth scales on the upper surface of the head, a larger mouth and smaller eyes, a narrower interorbital region, smaller average numbers of gill-rakers, a lower spinous dorsal fin, and generally darker color [25, 26]. Regarding habitat use, $P$. wiltoni is usually found in shallower waters than $P$. ramsayi $[25,26]$. A wider geographic sampling would be needed to explore the extent of genetic differentiation between these two species as well as possible adaptive differences or reinforcement mechanisms.

Table 2 Comparison of pairwise $F_{S T}$ between genomic and mitochondrial markers in pairs of closely related species

\begin{tabular}{lll}
\hline Pairwise $F_{\mathrm{ST}}$ & $\begin{array}{l}\text { Nuclear markers } \\
\text { (Loci) }\end{array}$ & $\begin{array}{l}\text { Mitochondrial } \\
\text { marker }\end{array}$ \\
\hline Species pair & $0.015(8849)$ & 0.63 \\
\hline P. ramsayi / P. wiltoni & $0.027(8540)$ & 0 \\
$\begin{array}{l}\text { P. guntheri / P. brevicauda } \\
\text { P. cornucola / P. cf. }\end{array}$ & $0.17(7307)$ & 0.78 \\
$\begin{array}{l}\text { cornucola } \\
\text { L. squamifrons / L. kempi }\end{array}$ & $0.037(9270)$ & 0.085 \\
\hline
\end{tabular}

The number of nuclear RAD loci used for the $F_{\mathrm{ST}}$ calculation is indicated in brackets
Both species belong to the longipes-species group within the genus Patagonotothen as defined by Balushkin (1976) [see also 12]. Two other species belonging to this group as well are (i) P. kreffti, which appears to be most closely related to $P$. ramsayi and is mainly differentiated in the length of the interorbital space [26], and (ii) $P$. longipes, which is closely related to $P$. wiltoni, differing mildly in the length of the pelvic fin [25, 26]. It has even been suggested that $P$. wiltoni and $P$. longipes should be treated as synonyms for the same species (Norman, 1937). Unfortunately, we were not able to include $P$. $k r e f f t i$ and $P$. longipes in our analysis. However, given the low degree of genetic differentiation between $P$. wiltoni and $P$. ramsayi, we would expect even weaker differentiation between $P$. wiltoni and $P$. longipes or between $P$. ramsayi and $P$. kreffti. Future investigations should include these species to shed light on their taxonomic status.

\section{The L. kempi - L. squamifrons species pair}

The mitochondrial locus failed to resolve taxonomic identity between these species, contrasting our results from the genome-wide RADseq analysis. Our results based on mtDNA are in line with those of Miya et al. 


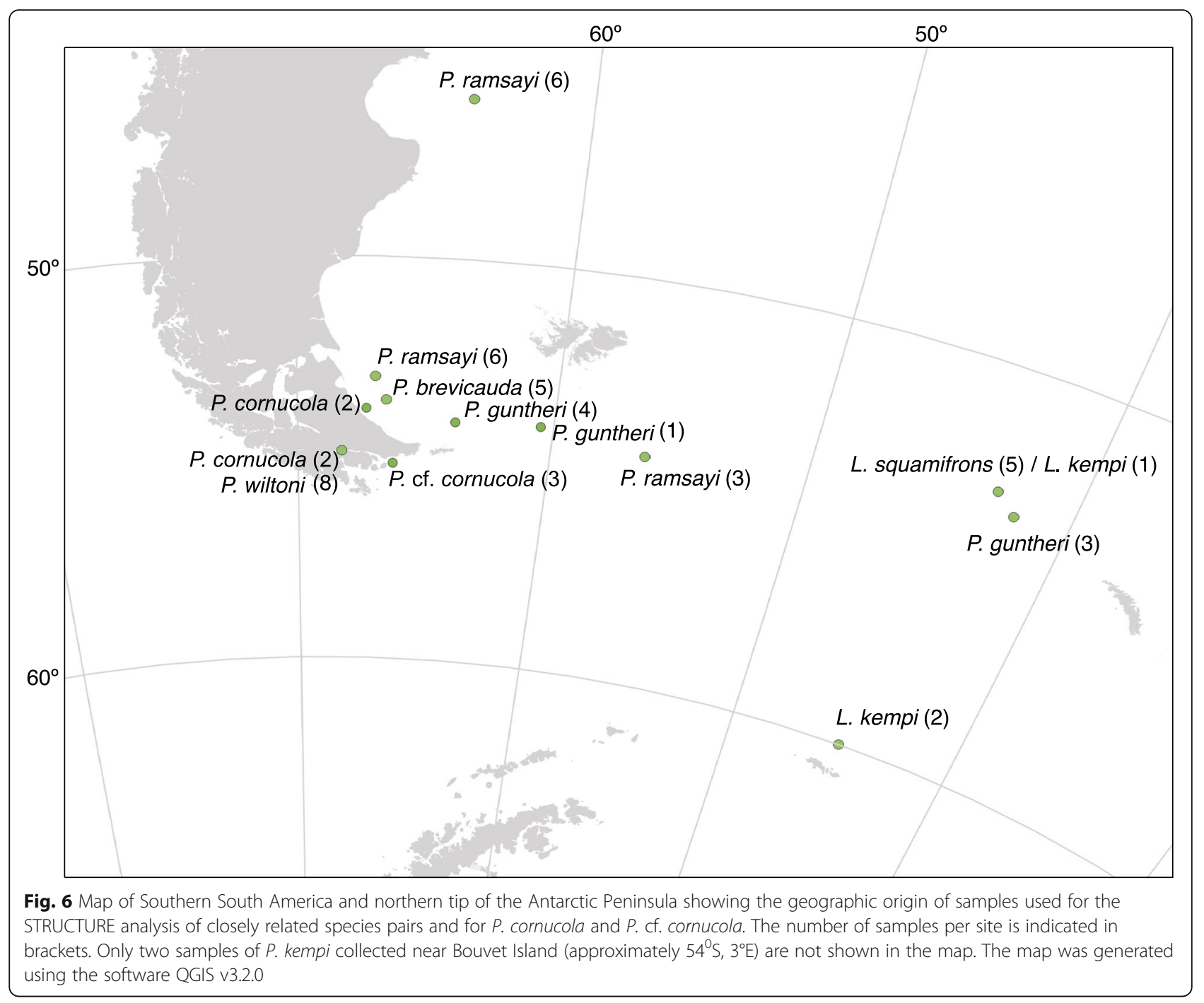

[39] who found low genetic differentiation between these two species based on mitochondrial markers. Furthermore, L. kempi specimens formed a clade nested within L. squamifrons in Miya et al. [39], which led the authors to propose that these may constitute geographic populations of the same species because the investigated $L$. kempi and $L$. squamifrons samples were obtained at sites separated by a large geographic distance. The same conclusion was reached by the study of Schneppenheim et al. [33] based on enzyme electrophoresis. In our analysis, two of the L. kempi specimens were collected near Bouvet Island, two near the South Orkneys, but one specimen was collected near Shag Rocks in exactly the same place as all $L$. squamifrons samples (Fig. 6). Therefore we can rule out the possibility that the differentiation found at genome-wide SNPs is simply due to isolation-by-distance. Our insights from nuclear SNPs are, despite their caveats due to small sample sizes, consistent with the hypothesis that $L$. kempi and $L$. squamifrons represent two separate species. The lack of differentiation found in mtDNA could be attributed to a low discriminating power of this single locus analysis. A wider geographic sampling, ideally including $L$. kempi and $L$. squamifrons individuals from the same geographic origin, and a genome-wide analysis will be needed to determine whether or not these two species should be lumped into one.

\section{Diversification timing and climatic history}

Our time-calibrated phylogeny suggests that the Patagonotothen radiation proceeded in a shorter period of time than previously thought ( $\sim 3$ versus 5 My respectively [6]). Furthermore, the lineages-through-time plot (Fig. $4 \mathrm{~b}$ ) indicates that the rate of species accumulation may not have been constant but rather stepwise. An early burst of diversification roughly coincides with the onset of the intensification of Quaternary glacial cycles about $2.5 \mathrm{Ma}$ [40]. A second round of speciation burst started around 1 Ma and coincides with the Greatest Patagonian Glaciation 
[41]. Finally, the three most closely related species pairs considered in our work $(P$. wiltoni $-P$. ramsayi, $P$. guntheri - P. brevicauda and L. kempi - L. squamifrons) show strikingly synchronous divergence times around 300 kya (Fig. 4). This dating falls within the Marine Isotope Stage 8 , a period characterized by global glacial advance that would have been particularly extensive in Patagonia [42]. Thus, according to our calibration, the main burst of diversification could be related to major climatic events in the region during the Quaternary, which would have shaped and probably promoted the Patagonotothen radiation. One possible mechanism that could have driven this diversification is isolation and differentiation into separate refugia followed by expansion and secondary contact. On the other hand, glaciation may have generally exacerbated the extinction rate, resulting in vacant niches that could have provided ecological opportunities for speciation. For example, glaciations are expected to affect inshore and coastal fauna to a greater extent while having a smaller effect on species that inhabit deeper waters. P. wiltoni, $P$. brevicauda and $P$. cornucola may therefore have originated from their deeper-living counterparts ( $P$. ramsayi, $P$. guntheri and $P$. cf. cornucola, respectively) following the ecological opportunities provided as a consequence of the retraction of glaciers in near shore or coastal areas. Whether the divergence was mainly promoted by geographic isolation during glaciation, by ecological opportunity following the retreat of glaciers, or by intrinsic biological factors remains an open question.

Recent studies have shown that per capita speciation rates are currently higher in the temperate and cold zone than the tropics whereas the rates were higher in the tropics in the past, but there is little insight into the underlying mechanisms of speciation $[43,44]$. The further study of the high latitude and recent radiation of Patagonotothen may contribute important data to understand how the latitudinal gradient in species diversity has developed and how it may evolve, a key frontier for future research in marine organisms [44].

\section{Conclusion}

This study represents the most extensive genome-wide phylogenetic analysis of the genus Patagonotothen. First, we demonstrate that RADseq is effective in gathering thousands of genome-wide markers to resolve phylogenetic relationships in the Antarctic notothenioid clade, including some of its oldest divergence events. We further show that the genetic boundaries between some species in the genus are diffuse, especially between $P$. brevicauda and $P$. guntheri, and between $P$. ramsayi and $P$. wiltoni. Some levels of recent introgression and/or insufficient time for fixation of alternative nuclear and mitochondrial alleles between the species may explain these results. We also provide genetic evidence for the presence of a new, potentially cryptic, Patagonotothen species. A deeper population-level sampling will be needed to refine these patterns and underlying processes in this ongoing radiation in which species are likely to have diverged very recently and may now be experiencing secondary contact.

Our time-calibrated phylogeny shows a period spanning more than one million years (representing more than a third of the age of the Patagonotothen clade) without species accumulation, suggesting that the rate of species accumulation was not constant but rather stepwise, probably shaped by major climatic events during the Quaternary. Future studies should address the underlying speciation mechanisms and the relative importance of putative allopatric divergence during glaciations and ecological opportunity afterwards.

Our phylogenetic analysis should serve as a reference for future investigations of the diversification and systematics within the genus. This first step in understanding the Patagonotothen radiation, a group of fish with Antarctic ancestry that have radiated in a sub-Antarctic environment, will also be important for insights into the possible fate of the spectacular diversity of Antarctic notothenioids in a warming Southern Ocean.

\section{Methods}

\section{Sampling and molecular methods}

In total, we collected 67 specimens belonging to twelve Patagonotothen species (Table 1) in southern Patagonia by means of three different methods: $(I)$ by bottom-trawling onboard the Oceanographic Vessel Puerto Deseado and the RV Polarstern, (II) by using trammel nets in the archipelago Islas Bridges at the Beagle Channel and (III) by capturing fish by hand in the intertidal zone during low tide in the Beagle Channel and in the Atlantic coast of Tierra del Fuego. When fishes were captured alive they were euthanized using buffered tricaine methanesulfonate (MS-222) or Eugenol (clove oil). Individuals were determined to the species level following Brickle et al. [24], originally adapted from Norman [25]. A piece of white muscle tissue was preserved from each individual in $90 \% \mathrm{EtOH}$ and stored at $-20{ }^{\circ} \mathrm{C}$ until subsequent analysis. In our analysis, we further included the following species as phylogenetic outgroups: Eleginops maclovinus, Harpagifer bispinis, Notothenia coriiceps, Lepidonotothen larseni, L. nudifrons, L. squamifrons and L. kempi, leading to a total of 96 individuals (Table 1).

Genomic DNA was extracted from about $20 \mathrm{mg}$ of muscle tissue using the DNeasy Blood and Tissue Kit (Qiagen). We then prepared three libraries of individually barcoded RAD [45], following the protocol described in Roesti et al. [46, 47], adopted from Hohenlohe et al. [48]. In short, each sample was individually subjected to 
restriction digestion using the $S b f 1$ enzyme, followed by the fusion of a specific 5-mer barcode to the restricted DNA of each individual. Subsequently no more than 40 individuals were pooled into a sequencing library. Two individual samples were included twice in different libraries for a posterior evaluation of the de novo assembly performance as suggested by Mastretta el al. [34]. Each library was single-end sequenced to $100 \mathrm{bp}$ reads in three separate lanes on an Illumina HiSeq2500 at the Quantitative Genomics Facility, D-BSSE, in Basel (Switzerland).

In addition, approximately $700 \mathrm{bp}$ of the mitochondrial cytochrome oxidase I (COI) gene were amplified by polymerase chain reaction (PCR) and subsequently Sanger-sequenced. For the PCR, we used the forward primer COX1L5928D 5'-TCRACYAAYCAYAAAGAYATYGGCAC-3' and the reverse primer COX1H6664D 5'-TAKACYTCWGGGTGDCCRAARAAYCA-3' in a volume of $30 \mu \mathrm{l}$, containing $15 \mu \mathrm{l}$ of total DNA $(4 \mu \mathrm{g} / \mathrm{ml})$, 1 unit of Taq DNA polymerase (Promega), $1 \mathrm{x}$ Taq polymerase buffer, dNTPs ( $0.2 \mathrm{mM}$ of each), forward and reverse primers $\left(0.3 \mathrm{mM}\right.$ of each) and $\mathrm{MgCl}_{2}(2.5 \mathrm{mM})$. The following cycling conditions were applied on a 2720 Thermal Cycler (Applied Biosystems): an initial denaturation of $3 \mathrm{~min}$ at $94^{\circ} \mathrm{C}, 35$ cycles of $30 \mathrm{~s}$ of denaturation at $94{ }^{\circ} \mathrm{C}, 30 \mathrm{~s}$ of annealing at $50^{\circ} \mathrm{C}$, and $1 \mathrm{~min}$ of extension at $72{ }^{\circ} \mathrm{C}$, followed by a final extension of $5 \mathrm{~min}$ at $72^{\circ} \mathrm{C}$. PCR products were then sequenced in both directions at Macrogen, South Korea. Chromatograms were scored and analyzed using BioEdit v7.2.5 [49].

\section{Assembly of reads into RAD loci and variant calling}

We conducted a de novo assembly of RAD loci with the software Stacks, version 1.41 [28]. Raw sequences were demultiplexed according to individual barcodes and filtered for low quality using the process_radtags module. From each sequence, we trimmed the 5-bp barcode and the remainder of the Sbf1 enzyme recognition site, yielding a final sequence read length of $89 \mathrm{bp}$. We then used the wrapper program denovo_map.pl to execute the components of the Stacks pipelines ustacks, cstacks and sstacks, followed by the rxstacks error correction module and, finally, a second iteration of cstacks and sstacks. The pipeline first aligns reads at the individual level and makes stacks with perfectly matching sequences when a minimum coverage is reached. This is controlled by the parameter $\mathrm{m}$ (minimum stack depth) that was set to 6 . Afterwards, based on the number of nucleotide differences between stacks, the software merges putative alleles together into a locus. This is controlled with parameter $M$ (distance allowed between stacks) that was set to $M=3$. Subsequently, a catalogue of all loci and alleles across individuals was generated and the software merged stacks up to a threshold distance that is controlled by the parameter $\mathrm{n}$, which was set to $\mathrm{n}=3$. Finally, we exported SNPs (including IUPAC encoded ambiguities for heterozygous sites), using the program populations from the Stacks pipeline. We excluded loci absent in more than $5 \%$ of the total samples and loci that did not reach a minimum coverage depth of 12 per individual.

During the preparation of the manuscript we became aware of the protocol suggested in reference [29] for parameters optimization in Stacks. Consequently, we followed this protocol to assess the performance of the parameters we have used (especially $M=3$ and $n=3$ as described above). We selected 10 samples, 2 from each of five species of the genus Patagonotothen that are representative of the variation within the genus $(P$. jordani, $P$. tessellata, $P$. cornucola, $P$. ramsayi and $P$. guntheri). We then iterated the denovo_map.pl module of Stacks varying $M$ from 1 to 6 and fixing $M=n$, and plotted the number of loci shared by at least $80 \%$ of the samples.

\section{Phylogenetic and population genomics analysis}

Maximum-likelihood trees were generated using RAxML (v8.2.1) [50] assuming a GTR + GAMMA model with the Lewis ascertainment bias correction on the CIPRES Science Gateway platform [51]. To account for possible incomplete lineage sorting in the relatively young radiation of Patagonotothen species, we also estimated the species tree with the software SNAPP [52]. We combined the Bayesian species-tree inference in SNAPP with divergence-time estimation based on the model of Stange et al. [53]. In brief, this model employs a strict molecular clock, links the population sizes of all extant and ancestral lineages, and assumes a Jukes-Cantor [54] model of sequence evolution. To time-calibrate the molecular clock, we applied log-normal age constraints according to the divergence times inferred by Colombo et al. [8], on the initial divergence of Trematomini (mean in real space: 6.0 Ma; standard deviation: $0.165 \mathrm{Ma}$ ) and on the most recent common ancestor of Lepidonotothen and Patagonotothen (mean in real space: $4.58 \mathrm{Ma}$; standard deviation: $0.25 \mathrm{Ma}$ ). To reduce computational cost, we performed the SNAPP analysis with a data set that contained no missing data and included only 3 samples per species and a single randomly selected SNP per RAD locus (see section 3.5). Input files for SNAPP were prepared with the script snapp_prep.rb (https://github.com/mmatschiner/snapp_prep). We performed four replicate SNAPP analyses with the same input file. Each replicate had a chain length of 1 million Markov-chain Monte Carlo (MCMC) iterations, of which the first 100,000 iterations were discarded as burnin. The samples generated by the four chains were merged into a single posterior distribution of parameter estimates and trees. A maximum-clade-credibility (MCC) tree was 
generated from the posterior tree sample using TreeAnnotator of the BEAST2 v2.4.3 software package [55].

We applied a population genomic approach to assess the level of differentiation between closely related species pairs within Patagonotothen using the Bayesian method of Pritchard et al. [56] implemented in the software STRUCTURE 2.3.4. Runs were performed with the number of genetic clusters $(K)$ fixed between 1 and 4 using an admixture model with correlated allele frequencies. Six independent runs were conducted for each $K$ value. Preliminary runs showed that convergence was achieved after 50,000 iterations. Thus, this was used as burn-in and the estimations were based on 100,000 additional iterations. We inferred the $K$ values that best captured the structure of the data by plotting the ' $\mathrm{Ln}$ Probability of Data' against $K$ as suggested by the software developers; however, more than one $K$ value were considered for subsequent analysis when biological meaningful information was observed. Results of STRUCTURE analyses were plotted with the script bar_plotter.rb (http:// evolution.unibas.ch/salzburger/software/bar_plotter.rb).

The genealogy of the mitochondrial locus was inferred using RAxML (v8.2.1) [50] assuming a GTR + GAMMA model on the CIPRES Science Gateway platform [51] and using a $\mathrm{NJ}$ algorithm as implemented in MEGA 6.0.6 [57]. A mitochondrial haplotype-genealogy graph was generated with Fitchi [58], using the phylogenetic tree obtained with RAxML as input.

\section{Additional files}

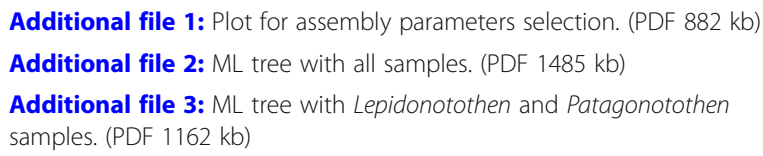

\section{Abbreviations}

AFGPs: Antifreeze glycoproteins; COI: Cytochrome Oxidase I; ILS: Incomplete Linage Sorting; ML: Maximum-Likelihood; NJ: Neighbour-Joining;

RADseq: Restriction site-associated DNA sequencing; SNPs: Single nucleotide polymorphisms

\section{Acknowledgements}

We would like to thank Mariela Victorio for field and laboratory assistance.

\section{Funding}

DAF and SGC were founded by the National Scientific and Technical Research Council from Argentina (CONICET) [grant PIP 0440, 2015]. WS acknowledges funding from the Swiss National Science Foundation (SNF) and the European Research Council (ERC). No funding body was involved in the design, collection, analysis, or interpretation of the data for this study, or in the writing of this manuscript.

\section{Availability of data and materials}

The data sets supporting the results of this article are available in the Dryad Digital Repository: https://doi.org/10.5061/dryad.c342rg7

\section{Authors' contributions}

SGC, DAF, and WS originally conceived and designed the study with substantial contributions from MR, MM, MD and RH. SGC, DAF, MM, MD, RH and WS contributed samples. SGC and MR conducted wet-lab work. SGC and MM performed the main genetic data analysis. All authors have made important intellectual contributions to the interpretation of results, manuscript preparation and finalization, and have read and approved the final version.

Ethics approval and consent to participate

All sampling procedures follow the guidelines of ethical use and care of animals in science approved by the Directive Board of the Southern Center of Scientific Research (CADIC-CONICET)(Act N ${ }^{\circ} 165,5 / 18 / 18$ ) and conform the proposals of the National Committee on Ethics in Science and Technology from Argentina (http://www.cecte.gov.ar/). The "Dirección General de Áreas Protegidas y Biodiversidad" from Tierra del Fuego granted the appropriated sampling permissions.

Consent for publication

Not applicable.

\section{Competing interests}

The authors declare that they have no competing interests.

\section{Publisher's Note}

Springer Nature remains neutral with regard to jurisdictional claims in published maps and institutional affiliations.

\section{Author details}

${ }^{1}$ Instituto de Ciencias Polares, Ambiente y Recursos Naturales (ICPA), Universidad Nacional de Tierra del Fuego (UNTDF), Fuegia Basket 251, V9410BXE Ushuaia, Argentina. ${ }^{2}$ Centro Austral de Investigaciones Científicas (CADIC-CONICET), Bernardo A Houssay 200, V9410BXE Ushuaia, Ushuaia, Tierra del Fuego, Argentina. ${ }^{3}$ Zoological Institute, University of Basel, Basel, Switzerland. ${ }^{4}$ Department of Zoology, University of British Columbia, Vancouver, BC, Canada. ${ }^{5}$ Department of Biosciences, Centre for Ecological and Evolutionary Synthesis (CEES), University of Oslo, Oslo, Norway. ${ }^{6}$ Forestry and Fisheries, Institute of Fisheries Ecology, Johann Heinrich von Thünen Institute, Federal Research Institute for Rural Areas, Hamburg, Germany.

Received: 26 July 2018 Accepted: 2 January 2019

Published online: 10 January 2019

\section{References}

1. Salzburger W. Understanding explosive diversification through cichlid fish genomics. Nat Rev Genet. 2018;19:705-17.

2. Eastman J. The nature of the diversity of Antarctic fishes. Polar Biol. 2005; 28(2):93-107.

3. Chenuil A, Saucède T, Hemery LG, Eléaume M, Féral J-P, Améziane N, et al. Understanding processes at the origin of species flocks with a focus on the marine Antarctic fauna. Biol Rev Blackwell Publishing Ltd. 2017;93:481-504.

4. Matschiner M, Colombo M, Damerau M, Ceballos S, Hanel R, Salzburger W. The adaptive radiation of notothenioid fishes in the waters of Antarctica. In: Riesch R, Plath M, Tobler M, editors. Extrem. Fishes: Springer; 2015. p. 35-57.

5. Matschiner M, Hanel R, Salzburger W. On the origin and trigger of the notothenioid adaptive radiation. PLoS One. 2011;6:e18911.

6. Near TJ, Dornburg A, Kuhn KL, Eastman JT, Pennington JN, Patarnello T, et al. Ancient climate change, antifreeze, and the evolutionary diversification of Antarctic fishes. Proc Natl Acad Sci. 2012;109:3434-9.

7. Cheng C-HC, Detrich HW. Molecular ecophysiology of Antarctic notothenioid fishes. Philos Trans R Soc Lond B Biol Sci. 2007;362:2215-32.

8. Colombo M, Damerau M, Hanel R, Salzburger W, Matschiner M. Diversity and disparity through time in the adaptive radiation of Antarctic notothenioid fishes. J Evol Biol. 2015;28:376-94.

9. Dornburg A, Federman S, Lamb AD, Jones CD, Near TJ. Cradles and museums of Antarctic teleost biodiversity. Nat Ecol Evol. 2017;1:1379-84. 
10. Collins MA, Shreeve RS, Fielding S, Thurston MH. Distribution, growth, diet and foraging behaviour of the yellow-fin notothen Patagonotothen guntheri (Norman) on the shag rocks shelf (Southern Ocean). J Fish Biol. 2008;72:271-86.

11. Jones CD, Eric Anderson M, Balushkin AV, Duhamel G, Eakin RR, Eastman JT, et al. Diversity, relative abundance, new locality records and population structure of Antarctic demersal fishes from the northern scotia arc islands and Bouvetøya. Polar Biol. 2008;31:1481-97.

12. Balushkin AV. Morphology, classification, and evolution of notothenioid fishes of the Southern Ocean (Notothenioidei, Perciformes ). J. Ichthyol. 2000:40:74-109.

13. Palma ED, Matano RP, Piola AR. A numerical study of the southwestern Atlantic shelf circulation: Stratified Ocean response to local and offshore forcing. J Geophys Res. 2008;113:C11010.

14. Cheng C-HC, Chen L, Near TJ, Jin Y. Functional antifreeze glycoprotein genes in temperate-water New Zealand nototheniid fish infer an Antarctic evolutionary origin. Mol Biol Evol. 2003;20:1897-908.

15. González-Wevar CA, Nakano T, Cañete JI, Poulin E. Concerted genetic morphological and ecological diversification in Nacella limpets in the Magellanic Province. Mol Ecol. 2011;20:1936-51.

16. Ceballos SG, Lessa EP, Victorio MF, Fernández DA. Phylogeography of the sub-Antarctic notothenioid fish Eleginops maclovinus: evidence of population expansion. Mar Biol. 2012;159:499-505.

17. Ceballos SG, Lessa EP, Licandeo R, Fernández DA. Genetic relationships between Atlantic and Pacific populations of the notothenioid fish Eleginops maclovinus: the footprints of quaternary glaciations in Patagonia. Heredity (Edinb). 2016;116:372-7.

18. Cousseau MB, Perrotta RG. Peces marinos de Argentina: biologia, distribucion, pesca. Mar del Plata: Publicaciones Espec. INIDEP; 2004.

19. Hüne M, Vega R. Feeding habits in two sympatric species of Notothenioidei, Patagonotothen cornucola and Harpagifer bispinis, in the Chilean Patagonian channels and fjords. Polar Biol. 2016;39:2253-62.

20. Laptikhovsky W. A comparative study of diet in three sympatric populations of Patagonotothen species (Pisces: Nototheniidae). Polar Biol. 2004;27:202-5.

21. Brickle P, Arkhipkin A, Shcherbich Z. Age and growth of a sub-Antarctic notothenioid, Patagonotothen ramsayi (Regan 1913), from the Falkland Islands. Polar Biol. 2006:29:633-9.

22. Laptikhovsky V, Arkhipkin Al, Brickle P. Life history, fishery, and stock conservation of the Patagonian toothfish around the Falkland Islands. J Fish Biol. 2006;49:587-94

23. Brickle P, Laptikhovsky V, Arkhipkin A, Portela J. Reproductive biology of Patagonotothen ramsayi (Regan, 1913) (Pisces: Nototheniidae) around the Falkland Islands. Polar Biol. 2006:29:570-80.

24. Brickle P, Shcherbich Z, Laptikhovsky V. Aspects of the biology of the Falkland's rockcod Patagonotothen ramsayi (Regan, 1913) on the southern Patagonian shelf. Sci Rep. 2005;81.

25. Norman JR. Coast fishes, Part II. The Patagonian Region. Discov Rep. 1937:16:1-150.

26. Balushkin A, Stehmann M. Results of the research cruises of FRV WaltherHerwing to South-America. LXXII. Patagonotothen kreffti sp. n., a new Patagonian notothen from Burdwood Bank, Western South Atlantic (Pisces, Perciformes, Nototheniidae). Arch Fisch Wiss. 1993:41(3):211.

27. Dettai A, Berkani M, Lautredou A-C, Couloux A, Lecointre G, Ozouf-Costaz C, et al. Tracking the elusive monophyly of nototheniid fishes (Teleostei) with multiple mitochondrial and nuclear markers. Mar Genomics. 2012;8:49-58.

28. Catchen J, Hohenlohe PA, Bassham S, Amores A, Cresko WA. Stacks: an analysis tool set for population genomics. Mol Ecol. 2013;22:3124-40.

29. Rochette NC, Catchen JM. Deriving genotypes from RAD-seq short-read data using stacks. Nat Protoc. 2017;12:2640-59.

30. Rutschmann S, Matschiner M, Damerau M, Muschick M, Lehmann MF, Hanel $R$, et al. Parallel ecological diversification in Antarctic notothenioid fishes as evidence for adaptive radiation. Mol Ecol. 2011;20:4707-21.

31. Richardson J. Ichthyology of the voyage of HMS Erebus \&amp. London: Terror, Under the Command of Captain Sir James Clark Ross; 1848.

32. DeWitt H, Heemstra P, Gon O. Nototheniidae. In: Gon O, Heemstra P, editors. Fishes South. Ocean. Grahamstown: JLB Smith Institute of Ichthyology; 1990. p. 279-380.

33. Schneppenheim R, Kock KH, Duhamel G, Janssen G. On the taxonomy of the Lepidonotothen squamifrons group (Pisces, Perciformes, Notothenioidei). Arch Fish Mar Res. 1994;42:137-48.

34. Mastretta-Yanes A, Arrigo N, Alvarez N, Jorgensen TH, Piñero D, Emerson BC. Restriction site-associated DNA sequencing, genotyping error estimation and de novo assembly optimization for population genetic inference. Mol Ecol Resour. 2015;15:28-41.

35. Valiñas MS, Helbling EW. Metabolic and behavioral responses of the reef fish Patagonotothen cornucola to ultraviolet radiation: Influence of the diet. J Exp Mar Bio Ecol Elsevier BV. 2016;474:180-4.

36. Pequeño G. Comments on fishes from the Diego Ramirez Islands, Chile. Japanese J. Ichthyol. 1986;32:440-2.

37. Fernández DA, Ceballos SG, Malanga G, Boy CC, Vanella FA. Buoyancy of sub-Antarctic notothenioids including the sister lineage of all other notothenioids (Bovichtidae). Polar Biol. 2011;35:99-106.

38. Dornburg A, Federman S, Eytan Rl, Near TJ. Cryptic species diversity in subAntarctic islands: a case study of Lepidonotothen. Mol Phylogenet Evol. 2016; 104:32-43.

39. Miya T, Gon O, Mwale M, Poulin E. Molecular systematics and taxonomic status of three latitudinally widespread nototheniid (Perciformes: Notothenioidei) fishes from the Southern Ocean. Zootaxa. 2016;4061:381-96.

40. Clapperton CM. Quaternary geology and geomorphology of South America. Amsterdam: Elsevier; 1993.

41. Rabassa J, Coronato A, Martínez O. Late Cenozoic glaciations in Patagonia and Tierra del Fuego: an updated review. Biol J Linn Soc. 2011;103:316-35.

42. Hein AS, Cogez A, Darvill CM, Mendelova M, Kaplan MR, Herman F, et al. Regional mid-Pleistocene glaciation in central Patagonia. Quat Sci Rev. 2017:164:77-94.

43. Schluter D. Speciation, ecological opportunity, and latitude. Am Nat. 2016; 187:1-18.

44. Rabosky DL, Chang J, Title PO, Cowman PF, Sallan L, Friedman M, et al. An inverse latitudinal gradient in speciation rate for marine fishes. Nature Springer US. 2018;559:392.

45. Baird NA, Etter PD, Atwood TS, Currey MC, Shiver AL, Lewis ZA, et al. Rapid SNP discovery and genetic mapping using sequenced RAD markers. PLoS One. 2008;3:e3376.

46. Roesti M, Moser D, Berner D. Recombination in the threespine stickleback genome-patterns and consequences. Mol Ecol. 2013;22:3014-27.

47. Roesti M, Hendry AP, Salzburger W, Berner D. Genome divergence during evolutionary diversification as revealed in replicate lake-stream stickleback population pairs. Mol Ecol. 2012;21:2852-62.

48. Hohenlohe PA, Bassham S, Etter PD, Stiffler N, Johnson EA, Cresko WA. Population genomics of parallel adaptation in threespine stickleback using sequenced RAD tags. PLoS Genet. 2010;6:e1000862.

49. Hall T. BioEdit: a user-friendly biological sequence alignment editor and analysis program for windows 95/98/NT. Nucl Acids Symp Ser. 1999;41:95-8.

50. Stamatakis A. RAxML version 8: a tool for phylogenetic analysis and postanalysis of large phylogenies. Bioinformatics. 2014;30:1312-3.

51. Miller MA, Pfeiffer W, Schwartz T. Creating the CIPRES Science Gateway for inference of large phylogenetic trees. 2010 Gatew. Comput. Environ. Work. GCE 2010. 2010.

52. Bryant D, Bouckaert R, Felsenstein J, Rosenberg NA, RoyChoudhury A. Inferring species srees directly from diallelic denetic karkers: bypassing gene trees in a full coalescent analysis. Mol Biol Evol. 2012;29:1917-32.

53. Stange M, Sánchez-Villagra MR, Salzburger W, Matschiner M. Bayesian divergence-time estimation with genome-wide SNP data of sea catfishes (Ariidae) supports Miocene closure of the Panamanian isthmus. Syst Biol Cold Spring Harbor Laboratory Press. 2018;67:681-99.

54. Jukes T, Cantor C. Evolution of protein molecules. In: Munro H, editor. Mamm. Protein Metab. New York: Academic Press; 1969. p. 21-132.

55. Bouckaert R, Heled J, Kühnert D, Vaughan T, Wu C-H, Xie D, et al. BEAST 2: a software platform for bayesian evolutionary analysis. PLoS Comput Biol. 2014;10:e1003537.

56. Pritchard JK, Stephens M, Donnelly P (2000) Inference of population structure using multilocus genotype data. Genetics 155:945-959.

57. Tamura K, Stecher G, Peterson D, Filipski A, Kumar S. MEGA6: molecular evolutionary genetics analysis version 6.0. Mol Biol Evolution. 2013;30: 2725-9.

58. Matschiner M. Fitchi: haplotype genealogy graphs based on the Fitch algorithm. Bioinformatics. 2016;32:1250-2. 